\title{
Spitzer view of Lyman break galaxies
}

\author{
Georgios E. Magdis ${ }^{1} \dagger$ and Dimitra Rigopoulou ${ }^{1}$ \\ ${ }^{1}$ Department of Astrophysics, University of Oxford, Keble Rd., Oxford, OX1 3RH, U.K. \\ email: dar@astro.ox.ac.uk,gem@astro.ox.ac.uk
}

\begin{abstract}
Using a combination of deep MID-IR observations obtained by IRAC, MIPS and IRS on board Spitzer we investigate the MID-IR properties of Lyman Break Galaxies (LBGs) at $\mathrm{z} \sim 3$, establish a better understanding of their nature and attempt a complete characterisation of the population. With deep mid-infrared and optical observations of $\sim 1000$ LBGs covered by IRAC/MIPS and from the ground respectively, we extend the spectral energy distributions (SEDs) of the LBGs to mid-infrared. Spitzer data reveal for the first time that the mid-infrared properties of the population are inhomogeneous ranging from those with marginal IRAC detections to those with bright rest-frame near-infrared colors and those detected at $24 \mu \mathrm{m}$ MIPS band revealing the newly discovered population of the Infrared Luminous Lyman Break Galaxies (ILLBGs). To investigate this diversity, we examine the photometric properties of the population and we use stellar population synthesis models to probe the stellar content of these galaxies. We find that a fraction of LBGs have very red colors and large estimated stellar masses $M_{*}>5 \times 10^{10} M_{\odot}$. We discuss the link between these LBGs and submm-luminous galaxies and we report the detection of rest frame 6.2 and $7.7 \mu \mathrm{m}$ emission features arising from Polycyclic Aromatic Hydrocarbons (PAH) in the Spitzer/IRS spectrum of an infrared-luminous Lyman break galaxy at $\mathrm{z}=3.01$.
\end{abstract}

\section{Introduction}

Observation and study of high-redshift galaxies is essential to constrain the history of galaxy evolution and give us a systematic and quantitative picture of galaxies in the early universe, an epoch of rigorous star and galaxy formation. Large samples of high-z galaxies that have recently become available, play a key role to that direction and have revealed a zoo of different galaxy populations at $\mathrm{z}$. There are various techniques for detecting high-z galaxies involving observations in wavelengths that span from optical to far-IR. Among the various methods the Lyman break dropout technique (Steidel \& Hamilton 1993), sensitive to the presence of the $912 \AA$ break, is designed to select $\mathrm{z} \sim 3$ galaxies. LBGs constitute at the moment the largest galaxy population at $\mathrm{z} \sim 3$ (Steidel et al. 2003). With observations spanning from X-rays (eg. Nandra et al. 2002) to near-infrared (Shapley et al. 2003) there has been a considerable progress into understanding the nature of population, but to fully characterize their properties (such as stellar mass, dust content, link to other $\mathrm{z} \sim 3$ populations) observations of longer wavelengths are required.

With the advent of Spitzer Space Telescope (Werner et al. 2004) we have access to longer wavelengths. IRAC bands $(3.6,4.5,5.8,8.0 \mu \mathrm{m})$ are crucial as they trace the restframe near infrared luminosities for galaxies at $0.5<\mathrm{z}<5$, (where the bulk of the stellar mass of a galaxy radiates) while $\operatorname{MIPS}(24,70,160 \mu \mathrm{m})$ and IRS $(5.3-40 \mu \mathrm{m})$ provide an insight into the interstellar medium of the population as they are sensitive to PAH features and dust re-radiation.

In this study we use IRAC and MIPS data covering 1000 and 244 LBGs respectively, lying on the fields Q1422+2309 (Q1422), DSF2237a,b (DSF), Q2233+1341 (Q2233),

$\dagger$ Present address: University of Oxford, DWB, Keble Rd., Oxford, OX1 3RH, U.K. 
SSA22a,b (SSA22), B20902+34 (B0902), QSOHS1700+6416 (Q1700), Extended Groth Strip (EGS) and Hubble Deep Field North (HDFN). Those LBGs have previously beeen identified from their optical colours by Steidel et al. (2003). In section 2 we search for mid-infrared counterparts of the LBGs, extend their SEDs to mid-infrared and investigate their mid-infrared colours as well as their physical properties such as stellar mass and dust content. In section 3 we examine the possible link between the IRAC/MIPS bright LBGs and the SMGs while in Section 4 using data obtained by IRS, we report the detection of $\mathrm{PAH}$ features arising from the mid-inrared spectrum of an ILLBG at $z=3.01$. In Section 5 we summurize the results of this Spitzer view of LBGs.

\section{Mid-infrared Properties of LBGs}

\subsection{The Spectral Energy Distridution of LBGs}

In a sample of 768 LBGs lying in the fields of our study (excluding EGS) we report the detection of 457, 456, 151 and 158 at 3.6, 4.5, 5.8 and $8.0 \mu \mathrm{m}$ IRAC bands respectively. Source extraction, photometric analysis, number counts and mid-infrared identification of LBGs are discussed in detail by Magdis et al. 2007 (in prep.). We extend the spectral energy distribution of the LBGs to rest frame near-infrared and improve dramatically our understanding of the nature of LBGs. Figure 1 shows the rest-UV/optical/near-infrared SEDs of all LBGs of the current sample with confirmed spectroscopic redshift. UV/optical data are obtained from Steidel et al. 2003, while IRAC data come from the present work. While the rest-UV/optical show little variation (2-3 magnitudes), the rest frame near infrared colour spread over 6 magnitudes. The addition of IRAC bands reveals for the first time that LBGs display a variety of colors and their rest-near-infrared properties are rather inhomogeneous, ranging from:

- Those that are bright in IRAC bands and exhibit $R-[3.6]>1.5$ colours. Their SEDs are rising steeply towards longer wavelengths and based on their $\mathrm{R}-[3.6]$ we call them "red" LBGs-z, to

- Those that are faint or not detected at all in IRAC bands with $R-[3.6]<1.5$ colour. Their SEDs are rather flat from the far-UV to the NIR with marginal IRAC detections and as they exhibit bluer $\mathrm{R}-[3.6]$ colours we call them "blue" LBGs-z.

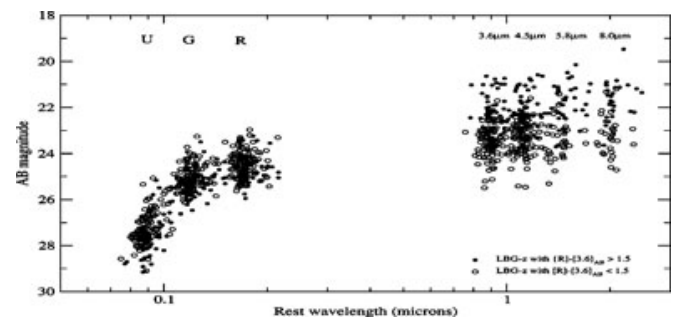

Figure 1. Spectral energy distribution for LBGs with spectroscopic redshift and classified as galaxies. Empty circle represent LBGs with $R-[3.6]<1.5$, while filled circle s represent LBGs with $R-[3.6]<1.5$, revealing that the rest-near-infrared of the population displays a wide range of colours

\subsection{The $8 \mu m$ sample, Stellar Masses and Dust Content}

From the whole sample of the detected at $3.6 \mu \mathrm{m}$ and $4.5 \mu \mathrm{m}$ LBGs, a fraction of about $40 \%$ are detected in longer wavelengths, creating the the sample of the $8 \mu \mathrm{m}$ LBGs. We find that LBGs with $8 \mu \mathrm{m}$ counterpart temd to exhibit redder $<R-[3.6]>$ colors when compared to those that are not detected at $8 \mu \mathrm{m}$ with a mean value of $1.91 \pm 0.16$ 


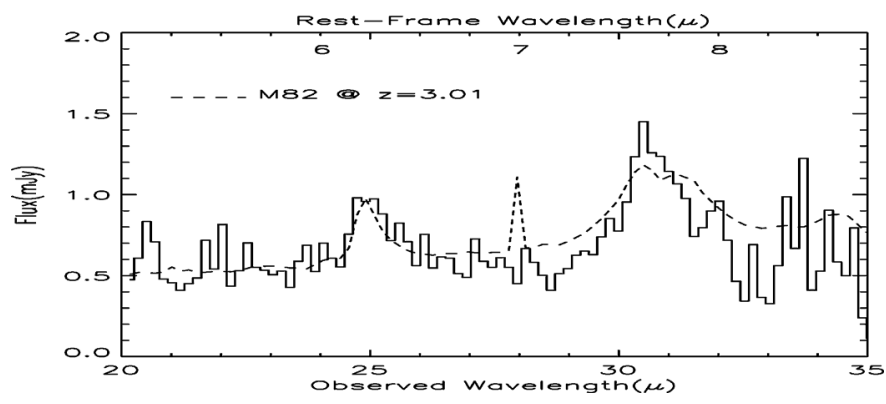

Figure 2. IRS spectrum of EGS20 J1418+5236. The dashed line is the M82 SED shifted to $z=3.01$. The spectrum has remarkably similar 6.2 and $7.7 \mu \mathrm{m}$ PAH emission-feature strength and shape to those of M82. We cross-correlated the IRS spectrum with that of M82 to derive a redshift of $z=3.01 \pm 0.016$.

and $1.02 \pm 0.26$ respectively. The significance of the $8 \mu \mathrm{m}$ sample is that for $\mathrm{z} \sim 3,8 \mu \mathrm{m}$ correspond to $\mathrm{K}$ rest-frame, sensitive to the bulk of the stellar emission of a galaxy and not only to the young population of a recent star-forming event. In the first study of Spitzer detection of LBGs in the EGS field (covering 244 LBGs), Rigopoulou et al. (2006) (R06 hereafter), using stellar synthesis population models generated with BC03, found that LBGs with $8 \mu \mathrm{m}$ are more massive dustier and relatively older when compared to the rest of the population. Preliminary results from our study (Magdis et al., in prep.), using both BC03 and CB07 (including an observationaly calibrated AGB phase) are in excellent agreement with R06. We find that LBGs with $8 \mathrm{mum}$ counterpart have estimated stellar masses of $\sim 8 \times 10^{10}$ and dust content of $\mathrm{E}(\mathrm{B}-\mathrm{V}) \sim 0.25$ while the mean values for the rest of the population are $2.7 \times 10^{10}$ and 0.18 respectively.

\section{ILLGS and the Link to SMGs}

From a sample of 244 LBGs in the EGS covered by MIPS, a fraction of about $\sim 5 \%$ where detected at $24 \mu \mathrm{m}$ creating the sample of the Infrared Luminous Lyman Break Galaxies (ILLBGs) sample (Huang et al. 2005). The detection of those LBGs at $24 \mu \mathrm{m}$ indicate the existance of significant amount of dust. R06 found the these LBGs have estimated stellar masses $M_{*}>10^{11} M_{\odot}$, ages $>1000$ Myrs and with exctinction that varies around $\mathrm{A}_{V} \sim 0.2$. These masses are similar to those of SMG as discussed by Borys et al. 2005. Also R06 found that ILLBGs show extreme R-K > 3 colors similar to the colors of SMGs examined by Chapman et al. (2000). Furthermore, ILLBGs and SMGs appear to have similar mid-infrared R-[3.6] and [8.0]-[24] colors (R06, Ashby et al. 2006). As these two population share many properties in common, it can be suggested that there must be a link between them. A possible scenario is one where SMGs and LBG form a continuum of objects with SMGs being the most dusty and most star-forming LBGs. In that case, ILLBGs should be at least detected in the submm bands. Therefore, we carried out submm observation of several ILLBGs with IRAM-MAMBO and we report the detection of 2 ILLBGs in the submm bands (Westphal-C47 $(4 \sigma)$ and Westphal-M30 $(3 \sigma))$. The data for the rest of the ILLBGs have not been analysed yet.

\section{IRS Spectroscopy of ILLBGs}

To further investigate the dust properties of the LBGs, we carried out follow up IRS mid-infrared spectroscopy of a number of ILLBGs from our sample. As preliminary result of this project, we report the detection of strong PAH features arising from the 
spectrum of the EGS21 ILLBG galaxy at $\mathrm{z}=3.01$ (Figure 2) (Huang et al. 2007). This is currently the highest redshift galaxy where these PAH emission features have been detected. The total infrared luminosity inferred from the MIPS $24 \mu \mathrm{m}$ and radio flux density is $2 \times 10^{13} L_{\odot}$, which qualifies this object as a so-called hyper-luminous infrared galaxy (HyLIRG). Given the strong PAH emission features and the lack of any evidence for an AGN, we conclude that star-formation dominates the emission from this $\mathrm{z}=3.01$ ILLBG.

\section{Conclusions}

The advent of Spitzer has dramatically improved our understanding of the LBGs. Using data obtained by IRAC/MIPS/IRS on board Spitzer we have reached the following conclusions for the population of the LBGS:

- IRAC colors have revealed the diversity of LBGs ranging from those with marginal detection in IRAC bands and R-[3.6] $<1.5$ colors, to those that have bright in IRAC bands and exhibit R-[3.6]>1.5 colors.

- LBGs detected at $8 \mu \mathrm{m}$ have redder R-[3.6] colors and on average are more massive, suffer more obscuration and have relatively older stellar populations when compared to the rest of the population.

- A fraction of about $\sim 5 \%$ of the LBGs do have dust as evidenced by MIPS $24 m u m$ detections, and are classified as ILLBGs. Those LBGs share many properties in common with the SMGs and preliminary results show that they can be detected at submm bands. It can therefore be suggested that a link between these two populations must exist.

- Strong PAH features arising from the mid-infrared spectrum of an ILLBG at $\mathrm{z}=3.01$ indicates the existence of dust in the interstellar medium of LBGs and suggest that the emission is dominated by star formation rather than an AGN.

\section{References}

Ashby, M. L. N., Dye, S., Huang, J.-S., Eales, S., Willner, S. P., Webb, T. M. A., Barmby, P., Rigopoulou, D., Egami, E., et al. 2006, ApJ 644, 778A

Borys, C.,Barnard, V., Bian, C., Blain, A. W., Brown, M. J. I., Dey, et al. 2005, ApJ 635, 853B

Chapman, S. C., Scott, D., Steidel, C. C., Borys, C., Halpern, M., Morris, S. L., et al. 2000, MNRAS 319, 318A

Huang, J.-S., Rigopoulou, D., Papovich, C., Ashby, M. L. N., Willner, S. P., et al. 2007, ApJ $660 \mathrm{~L}, 69 \mathrm{H}$

Huang, J.-S., Rigopoulou, D., Willner, S. P., Papovich, C., Shu, C., et al. 2005, ApJ 634, 137H

Nandra, K., Mushotzky, R. F., Arnaud, K., Steidel, C. C., et al. 2002, ApJ 576, 625N

Rigopoulou, D., Huang, J.-S., Papovich, C., Ashby, M. L. N., Barmby, P., Shu, C.; Bundy, K., Egami, E., Magdis, E. G., et al. 2006, ApJ 648, 81R

Shapley, A. E., Steidel, C. C., Pettini, M., \& Adelberger, K. L. 2003, ApJ 588, 65 S

Steidel, C. C. \& Hamilton, D. 1993, AJ 105, 2017S

Steidel, C. C., Adelberger, K. L., Shapley, A. E., Pettini, M., et al. 2003, ApJ 592, $728 \mathrm{~S}$

Werner, M. W., Roellig, T. L., Low, F. J., Rieke, G. H., Rieke, M., Hoffmann, W. F., Young, E., et al. 2004, ApJS154, 1W 